\title{
Colocalization of plaque macrophages and calcification in coronary plaques as detected by optical coherence tomography predicts cardiovascular outcome
}

\author{
Mathias Burgmaier ${ }^{1 *}$, Andrea Milzi ${ }^{1 *}$, Rosalia Dettori ${ }^{1 *}$, Kathrin Burgmaier ${ }^{2}$, \\ Martin Hellmich ${ }^{3}$, Mohammad Almalla ${ }^{1}$, Nikolaus Marx ${ }^{1}$, Sebastian Reith ${ }^{1}$ \\ ${ }^{1}$ Department of Cardiology, University Hospital of the RWTH Aachen, Germany \\ ${ }^{2}$ Department of Pediatrics, University Hospital of Cologne, Germany \\ ${ }^{3}$ Institute of Medical Statistics and Computational Biology (IMSB), University of Cologne, \\ Faculty of Medicine and University Hospital Cologne, Germany
}

Both plaque macrophage infiltration [1] and calcification [2] are recently suggested characteristics of plaque vulnerability in coronary lesions. Each of these two morphologic characteristics may foster the other $[3,4]$. Thus, the aim herein, is to evaluate this interdependence using optical coherence tomography (OCT), which, due to its supreme resolution, has the ability to detect both features. A recent study defined colocalization of macrophages and calcification (ColocCaMa) as a distance $<100 \mu \mathrm{m}$ between plaque macrophages and calcification. In this work an association was described between ColocCaMa and the more heavily calcified, but also less advanced and more vulnerable coronary lesions [5]. An example of ColocCaMa is shown in Figure 1A. In the present follow-up study, the aim was to investigate whether this more vulnerable plaque phenotype in the presence of ColoCaMa translates into more cardiovascular events.

One hundred and fifty five patients were prospectively enrolled, who underwent percutaneous coronary intervention (PCI) of the target lesion due to stable angina $(116,74.8 \%)$ or acute coronary syndrome (ACS; 39, 25.2\%) between 2012 and 2014 at the University Hospital of the RWTH Aachen, Germany. Patients were enrolled consecutively on days in which the study team was available. Further inclusion criteria were the suitability of the coronary target lesion for OCT analysis, as well as the presence of calcification within the target segment. A standardized follow-up was then performed with a median follow-up of 5.4 years (IQR 4.4-5.8). 25 patients were lost to follow-up, thus resulting in 130 patients with complete information. Death from any cause, new onset of myocardial infarction (MI), as well as emergent coronary revascularization and the composite endpoint of the three were noted. Written informed consent was obtained from all patients. The study was approved by the local ethics committee and conforms to the declaration of Helsinki. All statistical analyses were performed with SPSS (IBM Corp., Armonk, NY, USA).

Baseline age was $69.6 \pm 8.4$ years; $26(20 \%)$ patients suffered an ACS at inclusion. Prevalence of cardiovascular risk factors was high: $63.1 \%$ presented with diabetes, $85.4 \%$ hypertension and $62.3 \%$ hyperlipidemia. A thin-capped fibroatheroma was present in $34(26.1 \%)$ patients, macrophage infiltration in $48(36.9 \%)$ patients and ColocCaMa in $23(17.6 \%)$ patients. Frequency of thin-capped fibroatheroma was similar in patients with and without ColocCaMa (28 [26.2\%] vs. 6 [26.1\%], $\mathrm{p}=0.393)$. Inter- and intraobserver variability for the assessment of the presence of macrophages were 0.916 and 0.925 , respectively.

Address for correspondence: PD Dr. Sebastian Reith, MD, Department of Cardiology / Medical Clinic I, University Hospital of the RWTH Aachen, Pauwelsstr. 30, D-52074 Aachen, Germany, tel: ++49-241-80-36265, fax: ++49-241-80-33-36304, e-mail: sreith@ukaachen.de

Received: 14.01.2020 Accepted: 23.04.2020

*Mathias Burgmaier, Andrea Milzi und Rosalia Dettori contributed equally. 


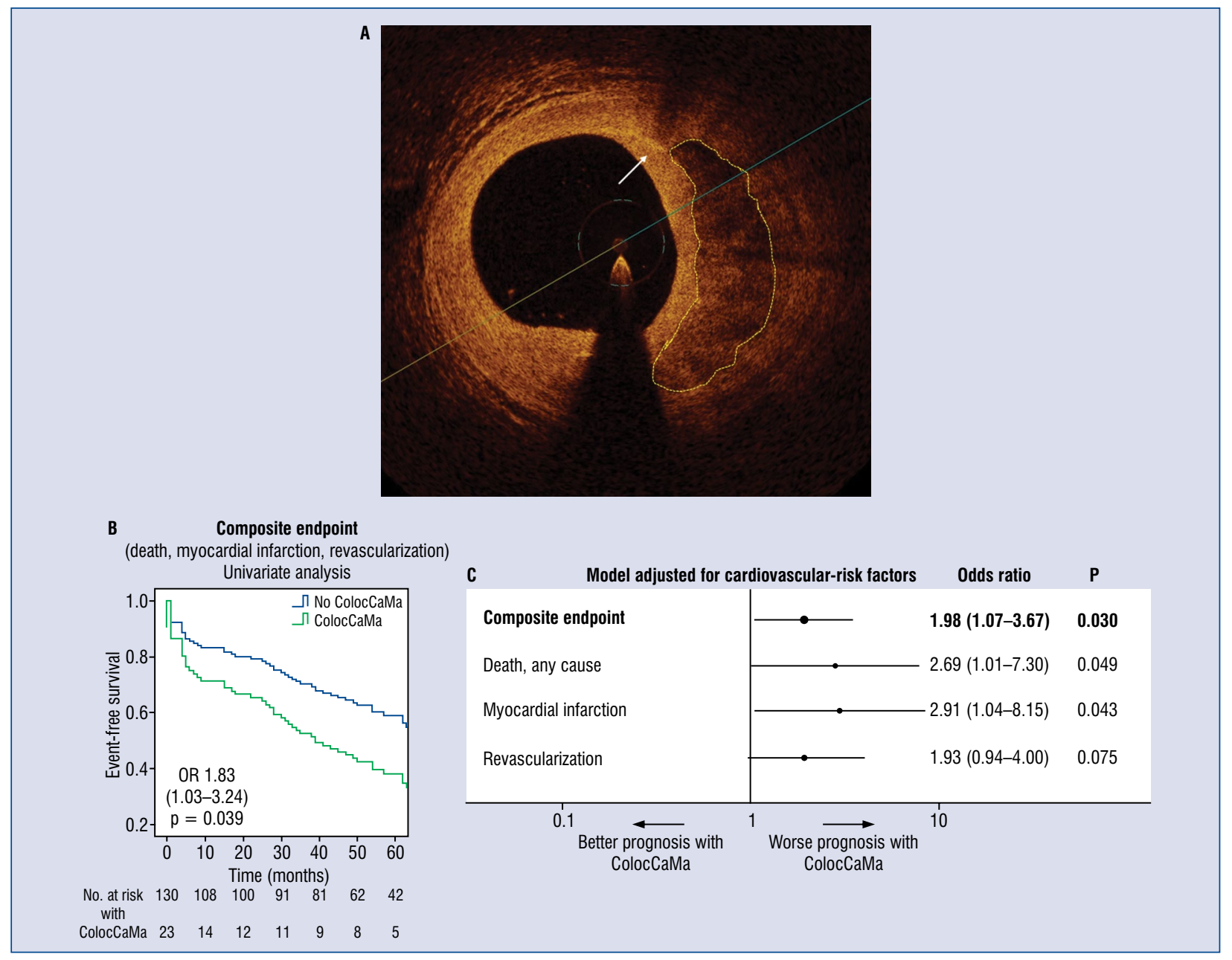

Figure 1. ColocCaMa in the coronary target/culprit lesion is associated with adverse cardiovascular outcome; A. A representative optical coherence tomography image of a ColocCaMa is shown; macrophage accumulation is marked with a white arrow, and calcification with a yellow border; B. The event-free curves derived from the Cox-regression show that in the presence of ColocCaMa (green line) in the target/culprit lesion, patients show a higher incidence of the composite endpoint of death from any cause, myocardial infarction and coronary revascularization; C. The forest-plot for single and composite endpoints is shown after adjustment for clinical presentation (acute coronary syndrome vs. stable angina), age, sex, glomerular filtration rate and relevant cardiovascular risk factors (body mass index, active nicotine use, presence of diabetes mellitus, hypertension, hyperlipidemia).

At follow-up, 29 (22.3\%) deaths, 26 (20\%) new MI and 43 (33.1\%) emergent coronary revascularizations were registered. The composite endpoint was met by $65(50 \%)$ patients. This high incidence of adverse cardiovascular events may be explained by the high cardiovascular risk profile of the enrolled population. No difference in the frequency of thin-capped fibroatheroma (20 [35.1\%] vs. 14 [28.6\%], $\mathrm{p}=0.617$ ) was detected among patients with or without adverse outcome at followup, whereas patients with adverse outcome showed more frequent plaque macrophage infiltration (32 [53.3\%] vs. 20 [32.3\%], p = 0.019). In contrast to a previous study [6], in-stent minimal lumen area $<4.5 \mathrm{~mm}^{2}$, narrowing of proximal or distal stent edges or distal dissection did not predict adverse outcome, probably due to the routine use of OCT-guided PCI-optimization. Using Coxregression analysis, patients with ColocCaMa showed a significantly higher incidence of the composite endpoint of death from any cause, MI and coronary revascularization (HR 1.83, 95\% CI 1.03-3.24, $\mathrm{p}=0.039$ ). In patients with ColocCaMa this increased risk for the composite endpoint is sustained over time and curves derived from Cox regression analysis are depicted in Figure 1B. Following adjustment for clinical presentation (ACS vs. stable angina), age, sex, glomerular filtra- 
tion rate and relevant cardiovascular risk factors (body mass index, active nicotine use, presence of diabetes mellitus, hypertension, hyperlipidemia) ColocCaMa still represented a significant risk factor for the composite endpoint (HR 1.98, 95\% CI 1.07-3.67, $\mathrm{p}=0.030$, Fig. $1 \mathrm{C}$ ). Furthermore, ColocCaMa was associated with death from any cause (HR 2.69, 95\% CI 1.01-7.30, $\mathrm{p}=0.049$ ) and MI (HR 2.91, 95\% CI 1.04-8.15, p = 0.043), whereas emergent coronary revascularization (HR $1.93,95 \%$ CI $0.94-4.00, \mathrm{p}=0.075$ ) did not reach significance in the adjusted model. Furthermore, in order to avoid any possible bias due to patients lost at follow-up, a multiple imputation analysis was performed, which confirmed the higher risk of the composite endpoint in patients with ColocCaMa (OR 1.78-1.82, $\mathrm{p}=0.039-0.046$ ).

The present group previously demonstrated that ColocCaMa is associated with a more vulnerable plaque phenotype [5]. In this study it was demonstrated that this translates into higher incidence of the composite endpoint of death from any cause, $\mathrm{MI}$ and coronary revascularization at follow-up.

Postinterventional OCT was performed in all patients, and relevant stent edge dissections or stent malappositions were treated immediately. Thus, it seems unlikely that the higher event rate in patients with ColocCaMa is caused by mechanical effects following coronary intervention. Furthermore, it has to be noticed that the survival curves progressively diverge over time suggesting an atherosclerosis-mediated effect. This is in line with the role of macrophages and microcalcifications as active players in atherogenesis and plaque destabilization. Even though stent implantation was performed in all the current patients, the data may be interpreted in light of the current concept of "vulnerable patient", i.e. patients with a tendency towards multifocal vulnerable lesions [7]. Specifically, previous OCT studies demonstrated more vulnerable lesions in non-culprit vessels of patients with ACS compared to stable coronary artery disease $[1,8]$. In this scenario, ColocCaMa may be a marker of patient vulnerability, thus unmasking the higher risk of certain sub-populations. On the other hand, ColocCaMa could also be associated with an accelerated local neo-atherosclerosis following stent implantation, which may therefore lead to re-stenosis and eventually to adverse outcomes. However, as standardized follow-up coronary angiographies were not performed in this study due to ethical reasons, it is still unclear if the composite endpoint is driven by a non-culprit lesion or culprit lesion stent failure due to ColocCaMa - this needs to be investigated in future studies.

Given that both plaque macrophages and microcalcifications increase plaque destabilization, it is tempting to speculate about a causal role of ColocCaMa in the genesis of plaque vulnerability and future cardiovascular events. However, due to the study design it remains unclear whether ColocCaMa is causally involved in plaque destabilization or merely reflects a localized or systemic vulnerable coronary artery disease. In the present study, there was not sufficient information on target lesion revascularization during follow-up. Moreover, although the inter- and intraobserver variability in detecting macrophage infiltration is acceptable and a recent study using directional atherectomy showed good accuracy of accepted OCT-criteria [9] in detecting macrophages [10], the presence of ColocCaMa was not directly validated using histopathology in this study due to the study design. A promising tool for macrophage detection may be offered by computer-based reconstructions, however, they were not used in this project. Furthermore, the small number of patients did not allow drawing definite conclusions about a possible correlation of other features of plaque vulnerability with adverse outcome.

In summary, the present data suggests and may allow others to identify a subgroup of patients with high cardiovascular risk and indicates Coloc$\mathrm{CaMa}$ to be a novel vulnerable plaque feature.

\section{Conflict of interest: None declared}

\section{References}

1. Kato K, Yonetsu T, Kim SJ, et al. Nonculprit plaques in patients with acute coronary syndromes have more vulnerable features compared with those with non-acute coronary syndromes: a 3-vessel optical coherence tomography study. Circ Cardiovasc Imaging. 2012; 5(4): 433-440, doi: 10.1161/CIRCIMAGING.112.973701, indexed in Pubmed: 22679059.

2. Reith S, Milzi A, Dettori R, et al. Predictors for target lesion microcalcifications in patients with stable coronary artery disease: an optical coherence tomography study. Clin Res Cardiol. 2018; 107(9): 763-771, doi: 10.1007/s00392-018-1243-1, indexed in Pubmed: 29654434.

3. Aikawa E, Nahrendorf M, Figueiredo JL, et al. Osteogenesis associates with inflammation in early-stage atherosclerosis evaluated by molecular imaging in vivo. Circulation. 2007; 116(24): 2841-2850, doi: 10.1161/CIRCULATIONAHA.107.732867, indexed in Pubmed: 18040026.

4. Nadra I, Mason JC, Philippidis P, et al. Proinflammatory activation of macrophages by basic calcium phosphate crystals via protein kinase $\mathrm{C}$ and MAP kinase pathways: a vicious cycle of inflammation and arterial calcification? Circ Res. 2005; 96(12): 
1248-1256, doi: 10.1161/01.RES.0000171451.88616.c2, indexed in Pubmed: 15905460.

5. Burgmaier M, Milzi A, Dettori R, et al. Co-localization of plaque macrophages with calcification is associated with a more vulnerable plaque phenotype and a greater calcification burden in coronary target segments as determined by OCT. PLoS One. 2018; 13(10): e0205984, doi: 10.1371/journal.pone.0205984, indexed in Pubmed: 30356326.

6. Prati F, Romagnoli E, La Manna A, et al. Long-term consequences of optical coherence tomography findings during percutaneous coronary intervention: the Centro Per La Lotta Contro Linfarto Optimization Of Percutaneous Coronary Intervention (CLI-OPCI) LATE study. EuroIntervention. 2018; 14(4): e443-e451, doi: 10.4244/EIJ-D-17-01111, indexed in Pubmed: 29633940.

7. Naghavi M, Libby P, Falk E, et al. From vulnerable plaque to vulnerable patient. A call for new definitions and risk assessment strategies: Part I. Circulation. 2003; 108(14): 1664-1672, doi: 10.1161/01.cir.0000087480.94275.97.
8. Vergallo R, Ren X, Yonetsu T, et al. Pancoronary plaque vulnerability in patients with acute coronary syndrome and ruptured culprit plaque: a 3-vessel optical coherence tomography study. Am Heart J. 2014; 167(1): 59-67, doi: 10.1016/j.ahj.2013.10.011, indexed in Pubmed: 24332143.

9. Tearney GJ, Regar E, Akasaka T, et al. Consensus standards for acquisition, measurement, and reporting of intravascular optical coherence tomography studies: a report from the International Working Group for Intravascular Optical Coherence Tomography Standardization and Validation. J Am Coll Cardiol. 2012; 59(12): 1058-1072, doi: 10.1016/j.jacc.2011.09.079, indexed in Pubmed: 22421299 .

10. Habara M, Otsuka F, Tsuchikane E, et al. In vivo tissue characterization of human atherosclerotic plaques by optical coherence tomography: A directional coronary atherectomy study with histopathologic confirmation. Int J Cardiol. 2018; 268: 1-10, doi: 10.1016/j.ijcard.2018.05.022, indexed in Pubmed: 29804698 